\title{
Salvage of Unilateral Complete Ear Amputation with Continuous Local Hyperbaric Oxygen, Platelet-Rich Plasma and Polydeoxyribonucleotide without Micro-Revascularization
}

\author{
Sang Keon Lee, Yoon Min Lim, Dae Hyun Lew, Seung Yong Song \\ Institute for Human Tissue Restoration, Department of Plastic and Reconstructive Surgery, Severance Hospital, Yonsei University College of \\ Medicine, Seoul, Korea
}

\begin{abstract}
In many cases of complete ear amputation, microvascular surgery is required for tissue perfusion and organ survival. However, microvascular reconstruction is not always feasible in the absence of suitable vessels. Here, we present the case of a 76-year-old man who underwent complete amputation of the left ear after a collapse at home because of cardiogenic syncope. He was treated with primary replantation and underwent a postoperative salvage course including continuous local hyperbaric oxygen therapy (HBOT), platelet-rich plasma (PRP) injections, and polydeoxyribonucleotide (PDRN) injections. The ear was almost completely salvaged, with a tiny eschar at the mid-scapha on both the anterior and posterior aspects. This case demonstrates the efficacy of local HBOT with PRP and PDRN injections.
\end{abstract}

Keywords Replantation / Salvage therapy / Hyperbaric oxygenation / Platelet rich plasma / Polydeoxyribonucleotide

\author{
Correspondence: Seungyong Song \\ Department of Plastic and \\ Reconstructive Surgery, Yonsei \\ University College of Medicine, \\ 50 Yonsei-ro, Seodaemun-gu, \\ Seoul 03722, Korea \\ Tel: $+82-2-2228-2225$ \\ Fax: +82-2-393-6947 \\ E-mail: pssysong@yuhs.ac
}

Received: 3 Mar 2017 • Revised: 25 May 2017 • Accepted: 31 May 2017

pISSN: 2234-6163 • elSSN: 2234-6171 • https://doi.org/10.5999/aps.2017.00451 • Arch Plast Surg 2017;44:554-558

\section{INTRODUCTION}

Replantation of a completely amputated ear without microsurgery is a possible reconstructive modality in the absence of proper vessels for microsurgical revascularization. However, simple replantation of a totally amputated ear poses risks of complications such as partial or complete composite tissue loss due to poor perfusion. With advances in adjunctive postoperative treatments, such as hyperbaric oxygen therapy (НBOT), aspirin, prostaglandin, dextran- 40 , and the application of leeches, reports have described improved survival in cases of nearcomplete ear amputation in which the ear was attached by a narrow skin bridge. However, in cases of complete amputation, it is rare for the ear to be salvaged without microvascular reconstruction. This report presents a case of nonmicrosurgical salvage of a completely amputated ear with local HBOT, plateletrich plasma (PRP) injections, and polydeoxyribonucleotide (PDRN) injections.

\section{CASE}

This study was reviewed and approved by the Yonsei University Medical Center Institutional Review Board (no. 201510026). The informed consent was waived.

A 76-year-old man presented to the emergency center approximately 1 hour after experiencing total left ear amputation. The 
injury was sustained when the patient collapsed in his home because of cardiogenic syncope, which he had experienced several times before. Fortunately, he had placed the amputated ear in a saline-filled plastic container. On physical examination under local anesthesia, the left ear was sagittally cut through the midportion of the pinna, including most of the scapha and the entire concha (Fig. 1), and proper vessels for microsurgical revascularization (with a caliber of $>0.5 \mathrm{~mm}$ ) were not seen in either the amputated ear or the cut portion of the ear. After informing the patient about the possibility of partial or total loss of a composite graft, the decision was made to attempt replantation with the planned postoperative salvage technique of continuous local HBOT. The amputated ear was minimally debrided and reattached to the original anatomical location as precisely as possible. A 4-0 Vicryl suture was used to attach the amputated carti-

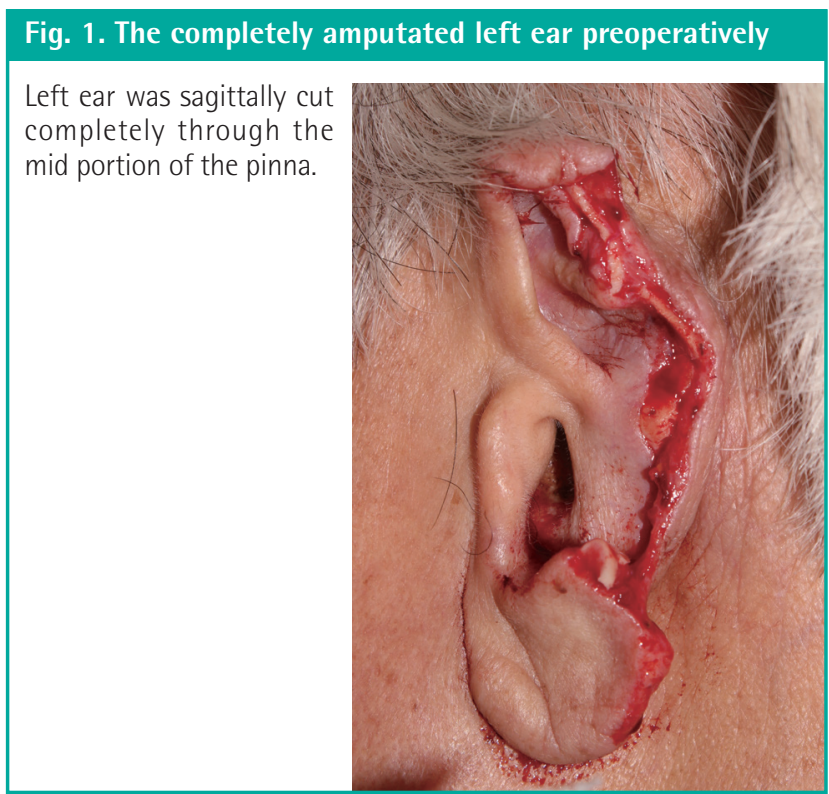

lage to the cartilage near the external auditory canal, and a 5-0 Vicryl suture was used to attach the subcutaneous layer of the amputated pinna. Meticulous 6-0 interrupted nylon sutures were then used to close the skin. After replantation, a gauze ball was packed into the ear canal, and the replanted ear was covered with a disposable paper cup for continuous local HBOT, instead of a specialized device (Fig. 2). The tailored-cut disposable paper cup was fixed to the skin surrounding the ear with surgical drape (Ioban 2, 3M, Hutchinson, Kansas, MO, USA) and the open-top area was covered with a transparent dressing material (Tegaderm film, 3M, Hutchinson) to prevent oxygen leakage. Finally, $100 \%$ oxygen from a generator was supplied to the paper cup chamber at $2 \mathrm{~L} / \mathrm{min}$ through a rubber tube. The positive pressure of oxygen on the replanted ear could be confirmed by bulging of the Tegaderm film that covered the paper cup chamber. The paper cup chamber with a surgical drape was replaced every 2 days to prevent the inevitable leakage caused by the pressure-induced detachment of the chamber from the skin. This continuous local HBOT began immediately after replantation and was continued for 24 hours a day. The patient was maintained on this HBOT regimen until 5 days before discharge, and had a total hospital stay of 19 days. On postoperative day (POD) 2, venous congestion of the whole replanted pinna was observed, and PRP and PDRN injections were initiated to increase tissue perfusion in the composite graft (Fig. 3). PRP was prepared by the following method: $30 \mathrm{~mL}$ of venous blood was drawn in acid citrate dextrose tubes, and then centrifuged at $150 \times \mathrm{g}$ for 15 minutes (the first spin). After discarding the lower portion corresponding to red blood cells, the supernatant was centrifuged again at $400 \times \mathrm{g}$ for 10 minutes (the second spin). Then, the lowest portion of corresponding platelet pellets was mixed with $1.5 \mathrm{~mL}$ of supernatant, making $2 \mathrm{~mL}$ of PRP. Fi-

\section{Fig. 2. Immediate postoperative photographs of the replanted ear}

(A) Anterolateral view. (B) Posterior view. (C) Paper cup for local hyperbaric oxygen therapy.
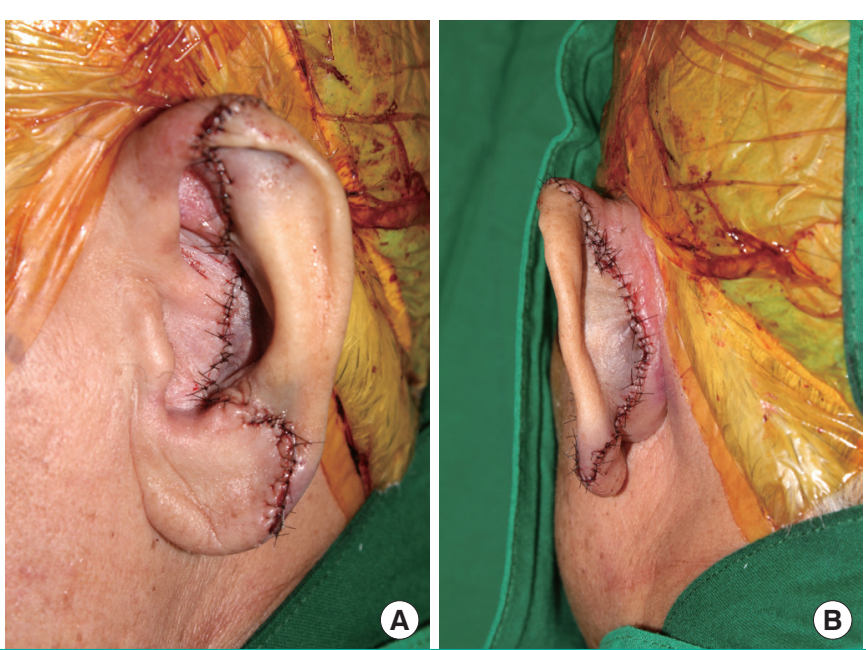


\section{Fig. 3. Postoperative day 2 photographs}

(A) Lateral view. (B) Posterior view.
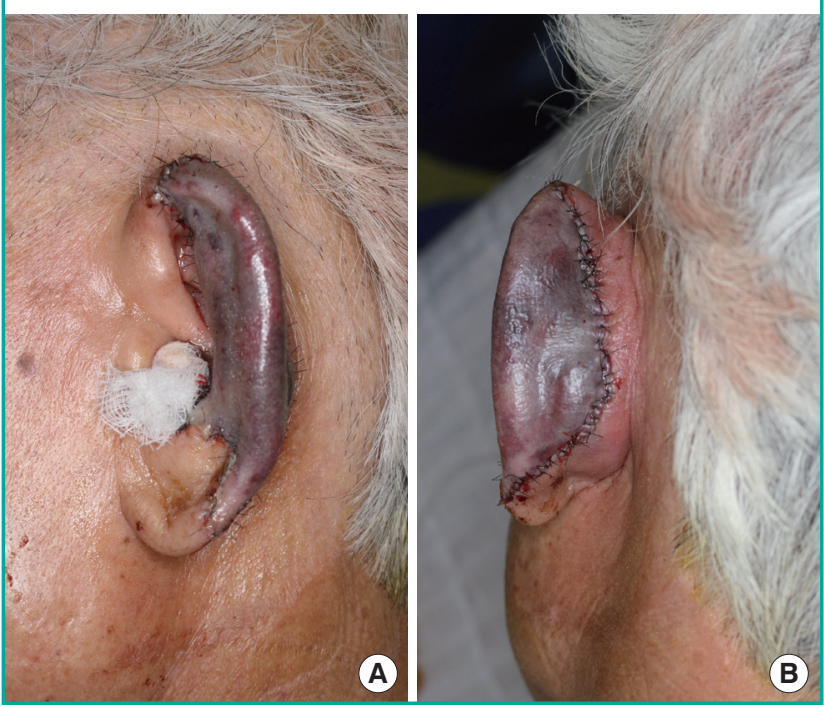

nally, $1 \mathrm{~mL}$ of $3 \%$ calcium chloride was added to the PRP to induce platelet activation. In addition, $3 \mathrm{~mL}$ of PRP was injected into the intact ear near the injury site (at 3 points, $1 \mathrm{~mL}$ at each point, $1 \mathrm{~cm}$ apart) every 3 days for 9 days, whereas PDRN (Placentex Integro, Mastelli Srl, Sanremo, Italy) was injected as 1 ample dose $(3 \mathrm{~mL}, 5.625 \mathrm{mg})$ on the replanted ear at 6 points evenly, every 2 days for 10 days. Marked improvement was seen by POD 9. On POD 14, the almost complete survival of the composite ear graft was observed, with 2 small areas of eschar on the mid-anterior scapha and the direct opposite side. Antibacterial ointment was applied to the eschar and it healed by secondary intention. On POD 18, exfoliation was found on the anterior and posterior mid-scapha where necrosis had previously been noted, and viable tissue was observed. The patient was discharged on POD 19 with instructions to maintain a sufficient application of antibiotic ointment to the wound. On POD 53, the ear was almost completely healed, with a tiny eschar at the mid-scapha in both the anterior and posterior aspects (Fig. 4).

\section{DISCUSSION}

Few reports have described the salvage of total ear amputation with nonmicrosurgical revascularization [1]. Here, we present a case in which it was possible to salvage and replant a completely amputated ear without micro-reanastomosis by implementing a postoperative salvage course including continuous local HBOT, PRP injections, and PDRN injections. In particular, PRP and PDRN injections have not been described in any previous reports of ear amputation cases.

Systemic HBOT has already been established as an adjunctive

\section{Fig. 4. Postoperative day 53 photographs}

(A) Anterolateral view. (B) Posterior view.
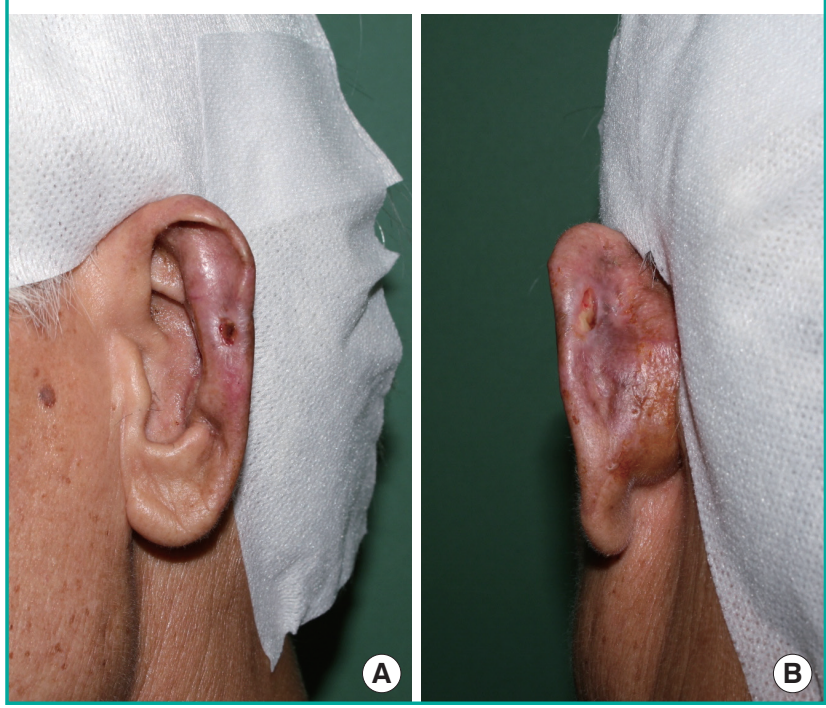

treatment to composite grafts for improving and supporting ischemic wounds [1,2], and Thom [3] described the mechanism of HBOT, reporting that HBOT increased cellular oxygen levels, which, in turn, increased the generation of reactive oxygen species and reactive nitrogen species, which served as signaling molecules in both angiogenesis and protection of ischemia-reperfusion injury pathways. Furthermore, based on these findings regarding HBOT, studies on local HBOT also have been conducted in animal models, and reports showing its efficacy have been published $[4,5]$. We used a disposable paper cup to create a local chamber for supporting hyperbaric oxygen continuously, at the rate of $2 \mathrm{~L} / \mathrm{min}$, for 24 hours a day (Fig. 2). Although it is not clear exactly what the atmospheric pressure was, we could confirm that a constant positive pressure was applied through a certain degree of bulging of the chamber lid, which was covered with Tegaderm. Although a certain amount of oxygen outflow was inevitable, the positive pressure could be maintained because the inflow more than compensated for the leakage. That is why we supplied $2 \mathrm{~L}$ of oxygen per minute. If a small amount of oxygen is introduced per minute, it is difficult to obtain sufficient positive pressure. On the other hand, if the amount of oxygen introduced per minute is too high, it is difficult to keep the chamber stable because of the high pressure. Although precise measurements and determinations of atmospheric pressure should be made in further studies, we believe that this technique supplied enough hyperbaric oxygen for the amputated ear to undergo neovascularization, and are confident that this information will be clinically helpful.

PRP injections for improving composite graft survival have been reported in rabbit models [6]. Jeon et al. [6] demonstrated 
significant increases in vascular endothelial growth factor (VEGF) expression in a PRP group compared with a control group (non-PRP injection). VEGF has already been reported to affect flap survival by promoting angiogenesis in a rat skin model [7], and it is considered that jointly with other growth factors such as fibroblast growth factor and platelet-derived growth factor stored in PRP, VEGF induces endothelial proliferation and neovascularization, thereby enhancing the blood supply of a composite graft. Therefore, the PRP injections are expected to have played an important role in improving the composite graft viability in this case. Moreover, research in other medical fields has established amounts of PRP that are clinically used in skin rejuvenation, hip or knee osteoarthritis, and tendinopathy [810]. However, no established data exist regarding the amount of PRP that should be used in flap injections, so the dose that we used in this case was based on the doses used in other clinical contexts.

PDRN is an adenosine A2 agonist that also induces VEGF and has been established to have multiple vascular actions, such as angiogenesis, in conditions of low tissue perfusion [11]. In the process of taking-up of composite grafts, inadequate tissue perfusion and ischemia-reperfusion injury are critical factors determining graft survival. The protective effect of VEGF on ischemia-reperfusion injury has been well established in transplantation surgery, such as kidney, lung and liver procedures [11-13], and those reports stated that VEGF affected endothelial cell survival, regulating their proliferation, regeneration, and apoptosis. Therefore, we expect that PDRN has the potential to improve the survival of composite grafts, enhancing neovascularization and protecting against ischemia-reperfusion injury by mediating an increase in VEGF. Lee et al. [14] likewise reported that PDRN injection increased VEGF expression in a rat skin flap model, and Kim et al. [15] reported that PDRN affected the healing process of chronic wounds, and introduced the quantity to be injected into the soft tissue that we used in our case.

In traumatic ear amputation, if microvascular anastomosis is considered difficult, the best treatment choice is usually delayed reconstruction with costal cartilage following primary closure, rather than simple replantation, due to the risk of loss of the composite graft. However, we propose an alternative method to the microvascular reconstruction of a completely amputated ear in the absence of suitable vessels, by using an immediate postoperative salvage course including continuous local HBOT, PRP injections, and PDRN injections, which support the marginally perfused composite graft through neovascularization and protect against ischemia-reperfusion injury. Although all these salvage techniques have been used in animal experiments, the rele- vant studies include poorly controlled samples, and no consensus exists regarding postoperative salvage care, the unexpectedly good cosmetic results in this case suggest that continuous local HBOT with PRP and PDRN injections may play a role in increasing the survival of composite grafts. To demonstrate the efficacy of these adjuvant salvage management methods, further prospective clinical studies should be conducted.

\section{CONFLICT OF INTEREST}

No potential conflict of interest relevant to this article was reported.

\section{PATIENT CONSENT}

The patient provided written informed consent for the publication and the use of their images.

\section{REFERENCES}

1. Kalus R. Successful bilateral composite ear reattachment. Plast Reconstr Surg Glob Open 2014;2:e174.

2. Fife CE, Eckert KA, Carter MJ. An update on the appropriate role for hyperbaric oxygen: indications and evidence. Plast Reconstr Surg 2016;138:107S-116S.

3. Thom SR. Hyperbaric oxygen: its mechanisms and efficacy. Plast Reconstr Surg 2011;127 Suppl 1:131S-141S.

4. Gordillo GM, Roy S, Khanna S, et al. Topical oxygen therapy induces vascular endothelial growth factor expression and improves closure of clinically presented chronic wounds. Clin Exp Pharmacol Physiol 2008;35:957-64.

5. Fries RB, Wallace WA, Roy S, et al. Dermal excisional wound healing in pigs following treatment with topically applied pure oxygen. Mutat Res 2005;579:172-81.

6. Jeon YR, Kang EH, Yang CE, et al. The effect of platelet-rich plasma on composite graft survival. Plast Reconstr Surg 2014;134:239-46.

7. Akimoto M, Takeda A, Matsushita O, et al. Effects of CBVEGF-A injection in rat flap models for improved survival. Plast Reconstr Surg 2013;131:717-25.

8. Gawdat HI, Tawdy AM, Hegazy RA, et al. Autologous platelet-rich plasma versus readymade growth factors in skin rejuvenation: a split face study. J Cosmet Dermatol 2017; 16:258-64.

9. Bennell KL, Hunter DJ, Paterson KL. Platelet-rich plasma for the management of hip and knee osteoarthritis. Curr Rheumatol Rep 2017;19:24.

10. Dallaudiere B, Pesquer L, Meyer P, et al. Intratendinous in- 
jection of platelet-rich plasma under US guidance to treat tendinopathy: a long-term pilot study. J Vasc Interv Radiol 2014;25:717-23.

11. Jeong EK, Jang HJ, Kim SS, et al. protective effect of polydeoxyribonucleotide against renal ischemia-reperfusion injury in mice. Transplant Proc 2016;48:1251-7.

12. Cao D, Wang M, Gong J, et al. Exogenous vascular endothelial growth factor delivery prior to endothelial precursor cell transplantation in orthotopic liver transplantation-induced hepatic ischemia/reperfusion injury. Liver Transpl 2017;23: 804-12.
13. Mittermayr R, Wolbank S, Redl H, et al. VEGF released from a fibrin biomatrix increases VEGFR-2 expression and improves early outcome after ischaemia-reperfusion injury. J Tissue Eng Regen Med 2017;11:2153-63.

14. Lee DW, Hong HJ, Roh H, et al. The Effect of polydeoxyribonucleotide on ischemic rat skin flap survival. Ann Plast Surg 2015;75:84-90.

15. Kim JY, Pak CS, Park JH, et al. Effects of polydeoxyribonucleotide in the treatment of pressure ulcers. J Korean Med Sci 2014;29 Suppl 3:S222-7. 